\title{
ANALYSIS OF ENERGY SPECTRA BY THE VORTEX METHOD WITH ARTIFICIAL TURBULENCE
}

\author{
Y. Ogami, \\ K. Nishiwaki, \\ and Y. Yoshihara \\ First, in order to use as an inlet condition for turbulent simulation, a method is presented \\ which produces numerically an artificial turbulence, namely, a series of velocity fluctuations \\ of which frequency is Gaussian, and energy spectrum and root mean square correspond to \\ Ritsumeikan University \\ Department of. Mechanical Engineering \\ 1-1-1 Noji-higashi, Kusatsu, Shiga \\ the given ones. Besides, the fluctuation data are determined by the characteristic parameters \\ of turbulent flows such as the inlet mean velocity, the kinematic viscosity, the Kolmogorov \\ scale and the integral time scale. Our examples show excellent accuracy and flexibility of \\ the method. Secondly, the vortex method has been studied to see the ability of the method \\ $525-8577$ \\ to deal with turbulent flows. It is found that the energy spectra produced by this agree well \\ with the ones given as the inlet condition, and that the vortex method is able to produce \\ ogmai@cfd.ritsumei.ac.jp \\ turbulent flows with the given parameters described above. \\ Key words: vortex method, artificial turbulence
}

\section{INTRODUCTION}

One of the crucial problems for the turbulent simulation is how to set up the inlet boundary condition that provides the physical quantities and qualities of turbulent flows as listed below.

1. Longitudinal and lateral spectra: $E_{L}(f)$ and $E_{T}(f)$

2. Root mean square of the velocity fluctuation:

$\mathrm{RMS}=\sqrt{\frac{\int_{0}^{T} U(t)^{2} d t}{T}}$

3. Mean velocity: $U$

4. Kinematic viscosity: $v$

5. Longitudinal and lateral integral scale: $L_{11}$ and $L_{22}$

6. Kolmogorov scale: $\eta=\left(\frac{2 v^{3} L_{11}}{u^{3}}\right)^{\frac{1}{4}}$

7. Gaussian frequency distribution of velocity fluctuations

Several researchers have presented numerical methods to produce velocity fluctuations for the turbulent simulation. Iwatani (1982) used the multidimensional autoregressive processes to produce velocity fluctuations from the power spectra and the cross spectra of fluctuations. The velocity fluctuations are given by a linear summation of white noise and of the past fluctuations with the coefficients that are obtained by solving a system of linear equations. The simulated results are somewhat noisy and have to be modified to obtain desired RMS.
Maruyama and Morikawa (1994), and Kondo et al. (1997) used the method of the trigonometric series with Gaussian random coefficients, in which the velocity fluctuations are expressed by a series of cosine and sine functions. The coefficients of the functions are obtained by solving a system of linear equations. They do not consider the distant grid points to lighten computational loads. This may produce numerical errors that cannot be disregarded.

The first purpose of this paper is to present a simpler and more accurate numerical method to produce series of velocity fluctuations. In this method, it is the longitudinal or lateral spectrum that is expressed by a series of cosine and sine functions. The coefficients of the functions are the velocity fluctuations themselves and these are obtained by solving a system of nonlinear (not linear) equations.

On the other hand, the vortex methods have been used for turbulent flow simulations with LES models (Leonard and Chua, 1989; Kiya et al., 1999; Mansfield et al., 1999; Kamemoto et al., 2000). Leonard and Chua (1989), and Kiya et al. (1999) incorporated the Smagorinsky model into the vortex methods by means of nonlinear corespreading algorithm. Mansfield et al. (1999) presented a LES scheme using a dynamic eddy diffusivity model. Kamemoto et al. (2000) reviewed the recent works on LES modeling and emphasized the necessity of developing wall turbulence models. To see if these models really work, it is necessary to examine whether the energy 


\section{CIÊNCIA/SCIENCE}

spectrum produced by these vortex methods are expected one because LES is to handle the energy spectrum of the lower frequency by modeling that of the higher frequency. Before doing this examination, it should be confirmed whether the vortex methods can handle the energy spectrum or are versatile enough to produce the prescribed energy spectrum. Totsuka and Obi (2000) calculated the energy spectrum using vortices and reported that the spectrum deviates from the target at the higher frequency regions when the resolution (vortex number) is insufficient.

The next purpose of this paper is to examine the capability of the vortex methods to produce flows with the prescribed physical quantities and qualities of turbulence mentioned at the beginning of this section. To do so, later in this paper we apply the results of next section to the vortex methods, and the LES model is used to see how it works.

\section{PRODUCING VELOCITY FLUCTUATION}

\section{One-Dimensional Case}

In this subsection, we introduce a method to numerically produce a series of velocity fluctuations, which are determined by the prescribed physical quantities or parameters mentioned earlier.

We consider the following longitudinal spectrum $E_{L}(f)$ and the Eulerian time-correlation $R_{E}(\tau)$,

$$
\left\{\begin{array}{l}
E_{L}(f)=\overline{4 u(t)^{2}} \int_{0}^{T} R_{E}(\tau) \cos (2 \pi f \tau) d \tau \\
R_{E}(\tau)=\frac{\overline{u(t) \cdot u(t+\tau)}}{\overline{u(t)^{2}}}
\end{array}\right.
$$

In this case, the directions of the mean velocity $U$ and the velocity fluctuation $u(t)$ are the same.

Equation can be rewritten as

$$
E_{L}(k)=\frac{4 T}{N^{2}}\left[\sum_{j=0}^{N-1} u_{j} \cos \left(2 \pi j \frac{k}{N}\right)\right]^{2}+\frac{4 T}{N^{2}}\left[\sum_{j=0}^{N-1} u_{j} \sin \left(2 \pi j \frac{k}{N}\right)\right]^{2}
$$

where the following relations have been employed.
Y. Ogami et al. Analysis of Energy Spectra by...

$$
\left\{\begin{array}{l}
\Delta t=\frac{T}{N}, \Delta f=\frac{1}{T}, t=j \Delta t=j \frac{T}{N}, \\
f=k \Delta f=\frac{k}{T}=\frac{k}{n \Delta t}, \\
f_{\max }=\frac{n}{2 T}=\frac{1}{2 \Delta t} \text { (Nyquist frequency) }
\end{array}\right.
$$

Equation is regarded as a system of simultaneous quadratic equations with $N$ unknowns, $u_{j}$. Since the number of the equations is $N / 2$ ( ), we divide Eq. into two parts to supply the deficit in the equations as

$$
\left\{\begin{array}{l}
\frac{T}{N} \sum_{j=0}^{N-1} u_{j} \cos \left(2 \pi j \frac{k}{N}\right)=\operatorname{sign}(r) \sqrt{\frac{T}{N} E_{L}(k) \frac{r^{2}}{r^{2}+1}} \\
\frac{T}{N} \sum_{j=0}^{N-1} u_{j} \sin \left(2 \pi j \frac{k}{N}\right)=\operatorname{sign}(r) \sqrt{\frac{T}{N} E_{L}(k) \frac{1}{r^{2}+1}}
\end{array}\right.
$$

where $r$ is a random number which we introduce expecting the frequency distribution of the solutions to be Gaussian. This division makes the equation number the same as the unknowns, and also linearizes the nonlinear equations, which promotes the convergence of the solutions.

When for the lower equation in Eq., all the coefficients in the left hand side become zero, which makes no sense. We use the following equation instead in order to incorporate RMS, which is given as one of the prescribed parameters.

$$
\mathrm{RMS}=\sqrt{\frac{\sum_{j=0}^{N-1} u_{j}^{2}}{N}}
$$

Equation is nonlinear and thus the system of the simultaneous equations has to be treated as a nonlinear system.

Even so, the velocity fluctuations can be obtained directly by as simple manner as merely solving these equations because the unknowns are the velocity fluctuations themselves unlike the methods mentioned earlier (Iwatani, 1982; Maruyama and Morikawa, 1994; Kondo et al., 1997).

It should be noted that the solutions to the system of equations and are not unique so that various sets of the solutions depending on the initial values and the random numbers can be obtained. 


\section{CIÊNCIA/SCIENCE}

This is another advantage of our method because of the coincidence with the characteristics of the turbulent flows.

\section{Three-Dimensional Case}

Since the inlet boundary is usually twodimensional, the series of velocity fluctuations passing through the boundary grids must be produced on the basis of both the longitudinal correlation and of the lateral correlation.

As shown in Fig.1, we consider that the inlet boundary is located on the $y-z$ plane, and that the fluid flows in the $x$-direction.

First, we obtain the velocity fluctuations that pass through the grid point $\left(y_{0}, z_{0}\right)$ by the method explained in the one-dimensional case section above. Then the fluctuations $u_{j}^{10}$ going through the next grid point $\left(y_{1}, z_{0}\right)$ can be also obtained by the same procedure except that the following lateral correlation has to be incorporated.

$$
\sum_{j=0}^{N-1} \frac{u_{j}^{00} u_{j}^{10}}{N}=\sum_{j=0}^{M / 2-1} \frac{1}{T} E_{T}(k) \cos \left(2 \pi \frac{k}{M}\right)
$$

where $E_{T}(k)$ is the energy spectrum of the lateral correlation, and $M$ is the grid number on the $y$ and $z$ - axes. Further, the fluctuations $u_{j}^{20}$ at the next point $\left(y_{2}, z_{0}\right)$ require the following two more equations.

$$
\begin{aligned}
& \sum_{j=0}^{N-1} \frac{u_{j}^{00} u_{j}^{20}}{N}=\sum_{j=0}^{M / 2-1} \frac{1}{T} E_{T}(k) \cos \left(2 \pi \frac{2 k}{M}\right) \\
& \sum_{j=0}^{N-1} \frac{u_{j}^{10} u_{j}^{20}}{N}=\sum_{j=0}^{M / 2-1} \frac{1}{T} E_{T}(k) \cos \left(2 \pi \frac{k}{M}\right)
\end{aligned}
$$

Generally, the fluctuations $u_{j}^{l m}$ at $\left(y_{l}, z_{m}\right)$ are calculated by Eqs. and, and the following equations

$$
\begin{array}{r}
\sum_{j=0}^{N-1} \frac{u_{j}^{l^{\prime} m} u_{j}^{l m}}{N}=\sum_{j=0}^{M / 2-1} \frac{1}{T} E_{T}(k) \cos \left(\begin{array}{c}
\left.2 \pi \frac{\left(l-l^{\prime}\right) k}{M}\right) \\
\left(0 \leq l^{\prime}<l\right)
\end{array}\right. \\
\sum_{j=0}^{N-1} \frac{u_{j}^{l m^{\prime}} u_{j}^{l m}}{N}=\sum_{j=0}^{M / 2-1} \frac{1}{T} E_{T}(k) \cos \left(\begin{array}{c}
\left.2 \pi \frac{\left(m-m^{\prime}\right) k}{M}\right) \\
\left(0 \leq m^{\prime}<m\right)
\end{array}\right.
\end{array}
$$

\section{Y. Ogami et al. Analysis of Energy Spectra by...}

The velocity fluctuations of the and components, can be obtained by the same procedures mentioned above except that the longitudinal correlation and the lateral correlation should be considered for each case (Tab. 1).

In this way, the velocity fluctuations on the inlet boundary are obtained from one grid to the next. The number of unknowns is always $\mathrm{N}$ regardless of the grid number $\mathrm{M}$, and these are easily obtained by solving a system of nonlinear equations. This effectively saves the computer memories and loads.

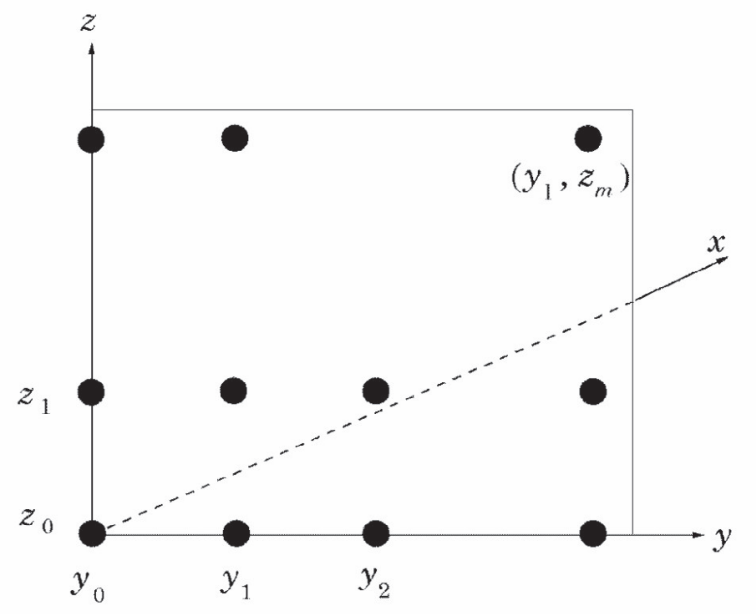

Figure 1 Three dimensional model.

Table 1. Each correlation to consider

\begin{tabular}{|c|c|c|c|}
\hline & $x$-direction & $y$-direction & $z$-direction \\
\hline$u$ & longitudinal & lateral & lateral \\
\hline$v$ & lateral & longitudinal & lateral \\
\hline$w$ & lateral & lateral & longitudinal \\
\hline
\end{tabular}

\section{Examples}

For example simulations are conducted using the parameters listed in Tab. 2. The longitudinal and lateral spectra given by Tennekes and Lumley (1999) are used as the target, and these spectra are later shown in Fig. 3 by the dashed line (longitudinal) and by the solid line (lateral). The system of nonlinear equations is solved by the subroutine "hybrd" provided in the free software package called minpac (downloadable at, for example, http://www.netlib.org/minpack/).

Figure 2 shows four examples of the simulated series of velocity fluctuations, which pass through the points $\left(y_{1}, z_{10}\right),\left(y_{2}, z_{10}\right),\left(y_{3}, z_{10}\right)$ and $\left(y_{4}, z_{10}\right)$. Though these are artificially produced, they resemble well those experimentally measured. 


\section{CIÊNCIA/SCIENCE}

Each series of the fluctuations is clearly different but the statistic is the same.

The spectra calculated from these fluctuations precisely agree with the target spectra as compared in Fig. 3. The relative error is less than $0.01 \%$, which can be controlled by the input parameter for "hybrd".

Figure 4 indicates that the velocity fluctuations are adequately random so that their frequency distribution fits the Gaussian distribution.

Table 2. Parameters used in simulation.

\begin{tabular}{|c|c|}
\hline Mean velocity: $U$ & $2.11 \mathrm{~m} / \mathrm{s}$ \\
\hline Kinematic viscosity: $v$ & $1.562 \times 10^{-5} \mathrm{~m}^{2} / \mathrm{s}$ \\
\hline $\begin{array}{c}\text { Root mean square of } \\
\text { velocity fluctuations: } r m s\end{array}$ & $0.159 \mathrm{~m} / \mathrm{s}$ \\
\hline Time step: $\Delta t$ & $1.94 \times 10^{-3} \mathrm{~s}$ \\
\hline $\begin{array}{c}\text { Grid spacing in flow } \\
\text { direction: } \Delta x\end{array}$ & $U t \Delta$ \\
\hline Integral scale: $L_{11}$ & $5 \Delta x$ \\
\hline Fluctuation number: $N$ & 200 \\
\hline $\begin{array}{c}\text { Grid number in } x \text { - and }-y \\
\text { directions: } M\end{array}$ & 20 \\
\hline
\end{tabular}

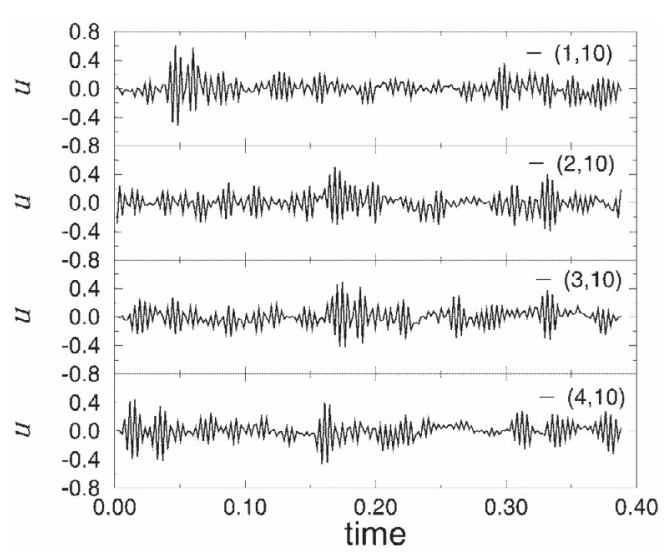

Figure 2. Calculated velocity fluctuations.

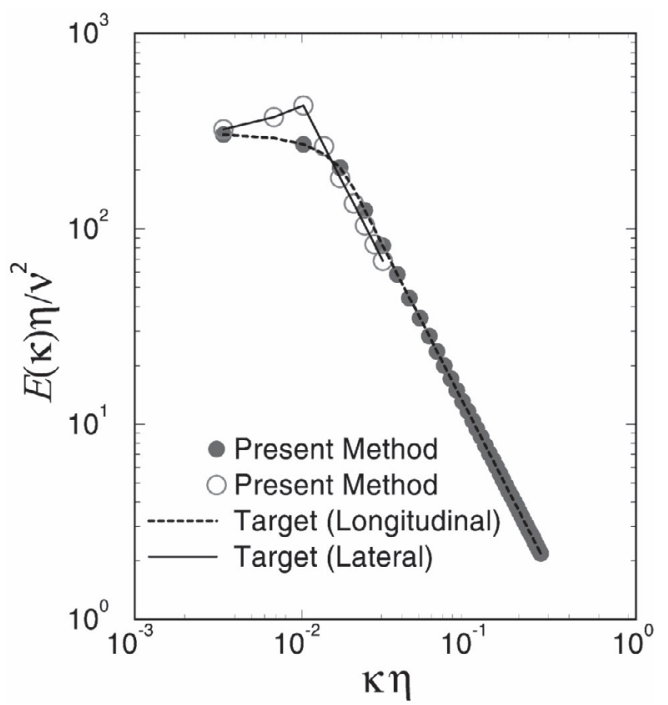

Figure 3. Target and calculated energy spectra.
Y. Ogami et al. Analysis of Energy Spectra by...

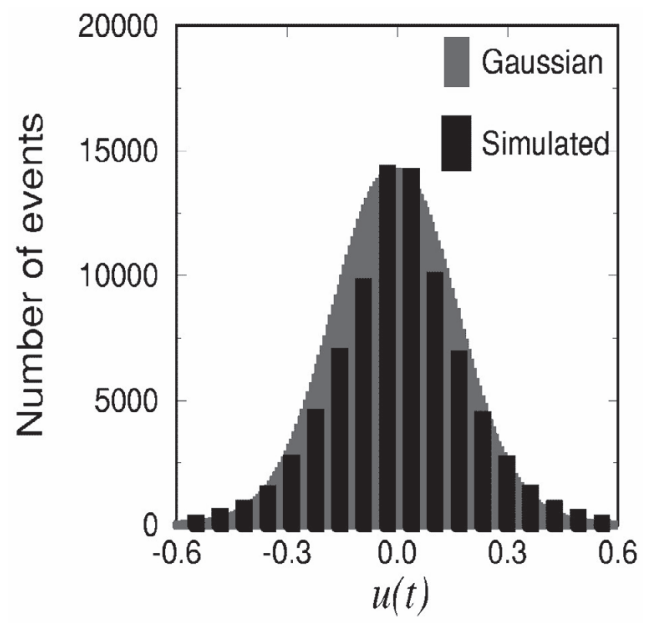

Figure 4. Frequency distribution.

\section{APPLICATION TO THE VORTEX METHOD}

In this section, the capability of the vortex methods to produce flows with the prescribed physical quantities and qualities of turbulence is examined. Also the LES model for the vortex method is studied.

\section{Vortex Strength}

The velocity fluctuations produced in the previous section can be used as the boundary conditions of the finite-difference methods as well as the vortex methods as explained below.

In the two dimensional flows, the vortex strength is given by

$$
\Gamma=\left(\frac{\partial v}{\partial x}-\frac{\partial u}{\partial y}\right) \Delta s
$$

where $\Delta s$ is the area the vortex occupies. Using the velocity fluctuations $u_{j}$ and $v_{j}$, Eq. can be approximately rewritten as

$$
\Gamma_{j h}=\left(\frac{v_{j+1, l}-v_{j-1, l}}{2 \Delta x}-\frac{u_{j, l+1}-u_{j, l-1}}{2 \Delta y}\right) \Delta s
$$

where the subscript indicates the vortex or velocity fluctuation passing through the point on the $y$-axis. Simulations are conducted for two different longitudinal integral scales, using the parameters listed in Tab. 3. The lateral spectrum is not considered for simplicity. The energy spectra obtained by these integral scales are illustrated in Fig. 5 showing that the energy at smaller wave numbers increases with $L_{11}$. 


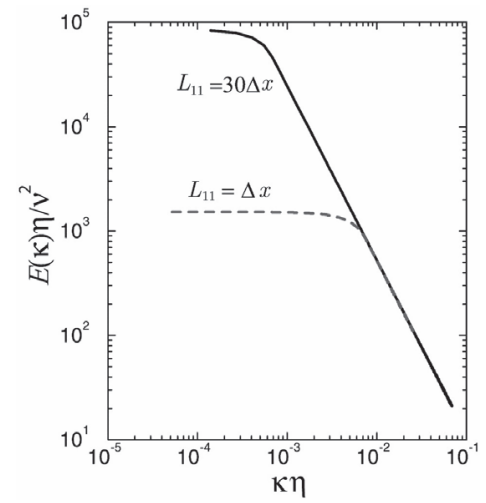

Figure 5. Target energy spectra.

Figures 6 and 7 show the velocity fluctuations respectively with $L_{11}=\Delta x$ and $30 \Delta x$ which are calculated by the method explained in the previous section. Roughly speaking, the fluctuations of lie in the straight band while these of in the wavy band, which indicates that the latter fluctuations have more energy in the smaller wave number regions as the target spectrum (Fig. 5).

The vortex strengths calculated using these fluctuations are shown in Fig. 8. The characteristics of the vortex strength are very similar to that of the velocity fluctuations.

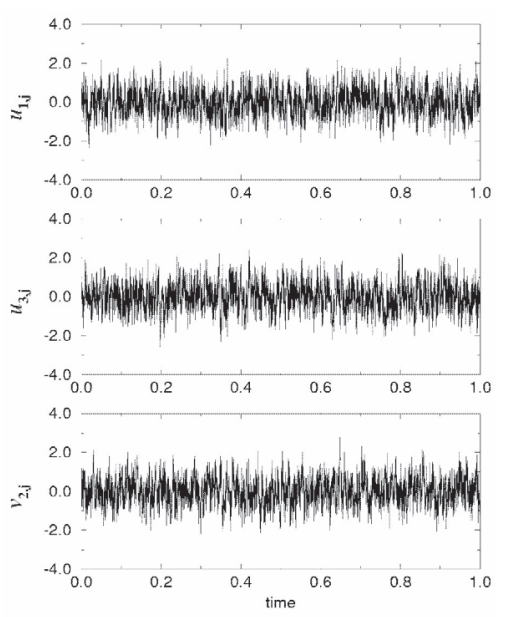

Figure 6. Velocity fluctuations at $L_{11}=\Delta x$.

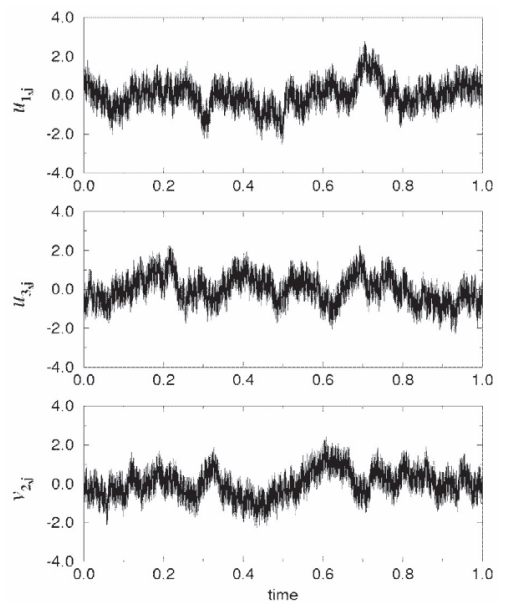

Figure 7. Velocity fluctuations at $L_{11}=30 \Delta x$.

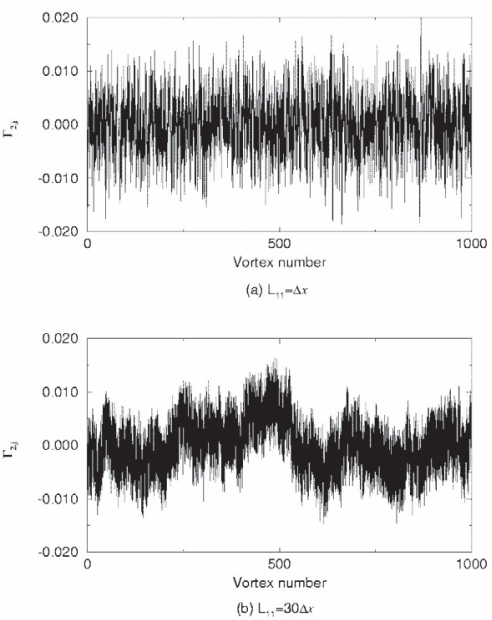

Figure 8. Vortex strength.

Table 3. Parameters used in simulation.

\begin{tabular}{|c|c|}
\hline Mean velocity: $U$ & $10 \mathrm{~m} / \mathrm{s}$ \\
\hline Kinematic viscosity: $v$ & $1.562 \times 10^{-5} \mathrm{~m}^{2} / \mathrm{s}$ \\
\hline $\begin{array}{c}\text { Root mean square of } \\
\text { velocity fluctuations: } \mathrm{rms}\end{array}$ & $1.0 \mathrm{~m} / \mathrm{s}$ \\
\hline Time step: $\Delta t$ & $8.2 \times 10^{-4} \mathrm{~s}$ \\
\hline $\begin{array}{c}\text { Grid spacing in flow } \\
\text { direction: } \Delta x\end{array}$ & $\Delta x$ and $30 \Delta x$ \\
\hline $\begin{array}{c}\text { Integral scale: } L_{11} \\
\text { Fluctuation number: } N\end{array}$ & 1024 \\
\hline $\begin{array}{c}\text { Grid number in } \\
\text { and }-z \text { directions: } M\end{array}$ & 2 and 1 \\
\hline
\end{tabular}

\section{Vortex Methods and Turbulence}

The vortices of the strengths calculated in the last subsection are supplied at the origin one by one at each time step. The series of the strengths are used repeatedly until the simulations stop. We do not expect that the field of the velocity fluctuations (Figs. 6 and 7) can be completely reproduced by these vortices because of the use of limited number of the vortices.

The movements of the vortices are calculated by the vortex method with the diffusion velocity (Ogami and Akamatsu (1991). The core radius of each vortex is updated to simulate the vortex stretch. The new core radius is $0.5 \times$ (distance to the nearest vortex). The parameters used for the simulations are listed in Tab. 4. 
Table 4. Parameters used in simulation.

\begin{tabular}{|c|c|}
\hline Mean velocity: $U$ & $10 \mathrm{~m} / \mathrm{s}$ \\
\hline Kinematic viscosity: $v$ & $1.562 \times 10^{-5} \mathrm{~m}^{2} / \mathrm{s}$ \\
\hline Time step: $\Delta t$ & $8.2 \times 10^{-4} \mathrm{~s}$ \\
\hline Initial core radius: $\sigma$ & $0.5 U t \Delta$ \\
\hline Updated core radius: $\sigma$, & $\begin{array}{c}0.5 \times(\text { distance to the } \\
\text { nearest vortex })\end{array}$ \\
\hline
\end{tabular}

Figure 9 shows the vortex distributions at time $5.03808 \mathrm{~s}$, namely after supplying six cycles of the series of vortices. The successive vortices are connected by the straight lines. This figure is extended four times in the direction. It is observed that the arrangement of the vortices with $L_{11}=30 \Delta x$, where $\Delta x=U \Delta t$, is much more wavy than the one with $L_{11}=\Delta x$ just like the velocity fluctuations and the vortex strengths.

Figure 10 shows the velocity fluctuations at the point $(20,0)$ produced by the vortices. The velocity is zero until the vortices reach this point at almost time $=2$.

The energy spectra calculated from these velocity fluctuations during $\mathrm{t}=4.1984 \sim 5.03808$ (namely, from 10245 steps to 10246 steps) are illustrated by the markin Figs. 11 and 12. The energy spectrum is multiplied bywhere is the total energy of the spectrum by the vortex method and is that of the target spectrum. Though the calculated values are scattered to some extent, they are distributed surely along the target spectra. The values of the limit for the high wave number, (averaged core radius), are also indicated by the dotted lines in Figs. 11 and 12. It is observed that the spectra larger than the limit $\left(4.11 \times 10^{-2}\right.$ for $L_{11}=\Delta x$ and $9.66 \times 10^{-2}$ for $\left.L_{11}=30 \Delta x\right)$ does not exist.
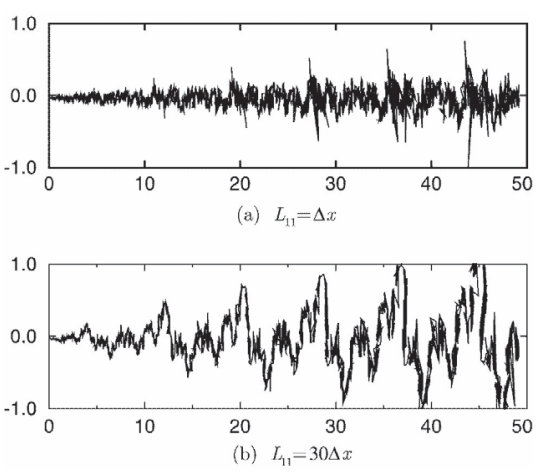

Figure 9. Vortex distributions.
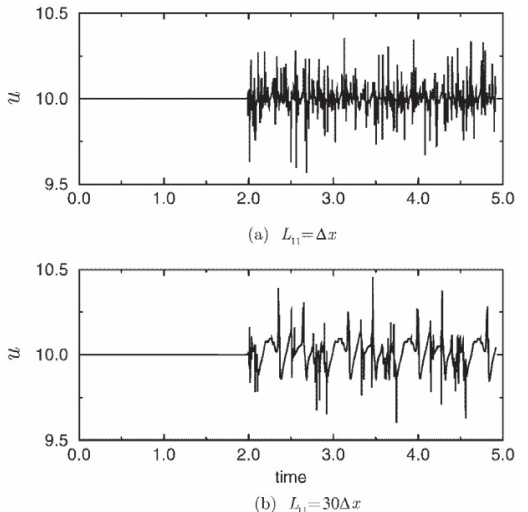

Figure 10. Velocity fluctuations produced by vortices.

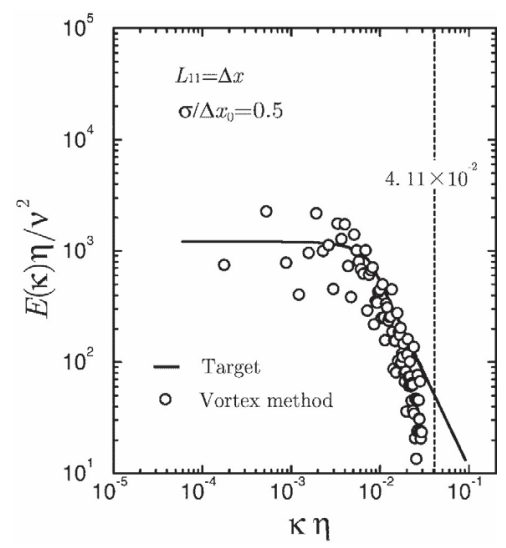

Figure 11. Energy spectra by the vortex method $\left(L_{11}=\Delta x\right)$.

\section{Vortex Methods and LES}

The LES model for the vortex method is used to see how it works. Simply, we add the subgrid scale viscosity

$$
v_{\mathrm{SGS}}=\max \left[0, C^{2} \sigma^{2} \frac{1}{\omega} \frac{d \omega}{d t}\right]
$$

adopted by Leonard and Chua (1989) to the diffusion velocity. Here, $C=0.17$ is employed.

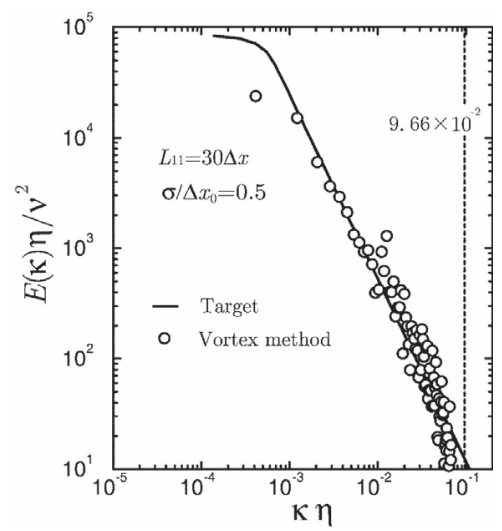

Figure 12. Energy spectra by the vortex method $\left(L_{11}=30 \Delta x\right)$. 


\section{CIÊNCIA/SCIENCE}

Even with the LES model, the spectrum obtained with $L_{11}=30 \Delta x$ is identical to the one without this model as shown in Fig. 13. This may be because the core radius is not large enough for the vortices to overlap each other with the initial core ratio $\sigma / \Delta x=0.5$, where $\Delta x$ is the initial vortex distance.

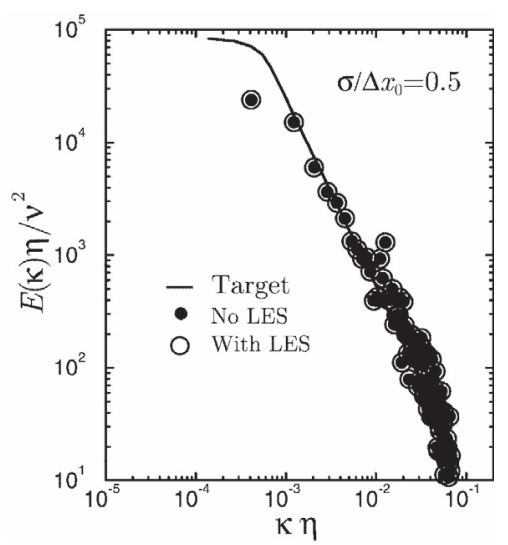

Figure 13. Energy spectra with and without LES model.

Figure 14 shows the comparison with the initial core ratio $\sigma / \Delta x=2$, where the vortices are more overlapped. The spectrum with the LES model is somewhat smaller at the higher wave number region than the one without the model because the energy is changed into the sub-grid scale viscosity. The wave number limit is $1.96 \times 10^{-2}$ for no LES case, and $1.92 \times 10^{-2}$ for LES case, which means that the averaged vortex core radius is smaller with LES model than that without the model. However, with the core ratio $\sigma / \Delta x=4$, in Fig. 15, this energy decrease is negligibly small.

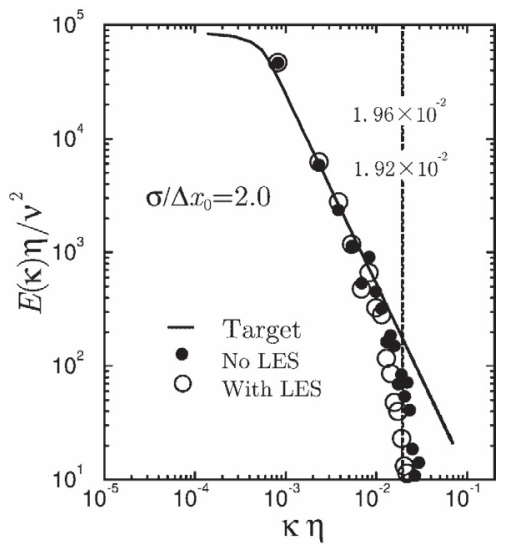

Figure 14. Energy spectra with and without LES model $\left(L_{11}=30 \Delta x, \sigma / \Delta x=2.0\right)$.
Y. Ogami et al. Analysis of Energy Spectra by...

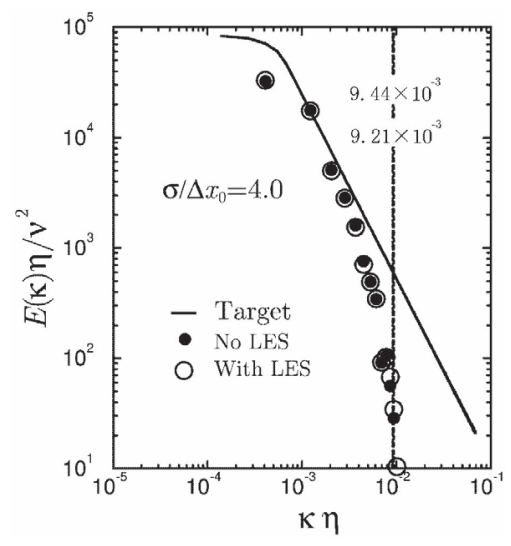

Figure 15. Energy spectra with and without LES $\operatorname{model}\left(L_{11}=30 \Delta x, \sigma / \Delta x=4.0\right)$.

With the integral scale $L_{11}=\Delta x$, the spectra obtained using the LES model are almost identical to the ones without the model as shown in Figs. 16-18. The limit of higher wave number is listed in Tabs. 5 and 6.

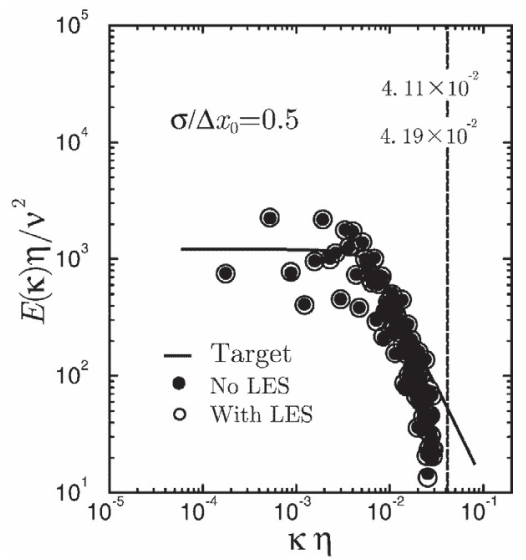

Figure 16. Energy spectra with and without LES model $\left(L_{11}=\Delta x, \sigma / \Delta x=0.5\right)$.

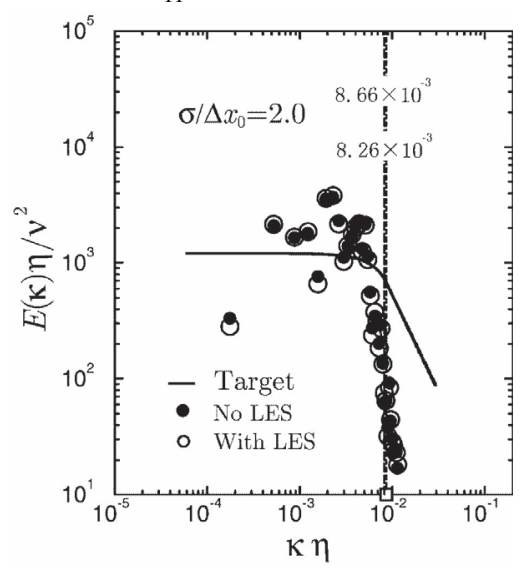

Figure 17. Energy spectra with and without LES $\operatorname{model}\left(L_{11}=\Delta x, \sigma / \Delta x=2.0\right)$.

Generally, the results with and without the LES model are very close, and the way to make use of the LES method for the vortex method will have to be studied. 


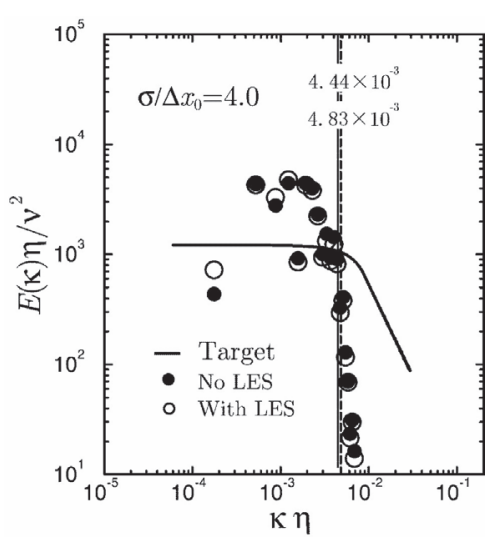

Figure 18. Energy spectra with and without LES $\operatorname{model}\left(L_{11}=\Delta x, \sigma / \Delta x=4.0\right)$.

Table 5. The limit of wave number $\pi / \bar{\sigma}$ with $L_{11}=30 \Delta x$.

\begin{tabular}{|c|cc}
\hline$\sigma / \Delta x$ & Without LES & With LES \\
\hline 0.5 & $9.66 \times 10^{-2}$ & $9.60 \times 10^{-2}$ \\
2.0 & $1.96 \times 10^{-2}$ & $1.92 \times 10^{-2}$ \\
4.0 & $9.21 \times 10^{-3}$ & $9.44 \times 10^{-3}$ \\
\hline
\end{tabular}

Table 6. The limit of wave number $\pi / \bar{\sigma}$ with $L_{11}=\Delta x$.

\begin{tabular}{|l|cl}
\hline$\sigma / \Delta x$ & Without LES & With LES \\
\hline 0.5 & $4.19 \times 10^{-2}$ & $4.11 \times 10^{-2}$ \\
2.0 & $8.66 \times 10^{-3}$ & $8.26 \times 10^{-3}$ \\
4.0 & $4.44 \times 10^{-3}$ & $4.83 \times 10^{-3}$ \\
\hline
\end{tabular}

\section{CONCLUSIONS}

First, a simple and accurate numerical method is presented to produce velocity fluctuations that are determined by the prescribed physical quantities and qualities of turbulence. The fluctuations are easily obtained by solving a system of nonlinear equations using free software. Also this method requires as many computer memories and computations as one-dimensional case even for the three dimensional calculations. The calculated energy spectra are quite accurate with less than $0.01 \%$ relative errors to the prescribed spectra.

Next, these fluctuations are used to examine the capability of the vortex methods to produce turbulent flows with the prescribed parameters. Although the energy spectra by the vortex method scatter to some extent, they are distributed along the prescribed spectra even at the higher frequency regions. It can be said that the vortex methods are able to simulate the target turbulence fairly well.

The results with the LES model are close to the ones without the model. For further work, the way to make use of the LES method for the vortex method will have to be studied.

\section{REFERENCES}

Iwatani, Y., 1982, Simulation of multidimensional wind fluctuations having any arbitrary power spectra and cross spectra, Journal of Wind Engineering, No.11, pp.5-18. (in Japanese)

Kamemoto, K., Zhu, B. and Ojima, A., 2000, Attractive features of an advanced vortex method and its subjects as a tool of lagrangian LES, Proceedings of $14^{\text {th }}$ Symposium of Computational Fluid Dynamics, B06-4.

Kiya, M., Izawa, S. and Ishikawa, H., 1999, Vortex method simulations of forced, impulsively started round jet, Proceedings of FEDSM'99, FEDSM99-6813.

Kondo, K. Murakami, S. and Mochida, A., 1997, Generation of velocity fluctuations for inflow boundary condition of LES, Journal of Wind Engineering and Industrial Aerodynamics, Vol.67\&68, pp.51-64.

Leonard, A. and Chua, K., 1989, Threedimensional interactions of vortex tubes, Physica D, Vol.37, pp.490-496.

Mansfield, J. R., Knio, O. M. and Meneveau, C., 1999, Dynamic LES of colliding vortex rings using a $3 \mathrm{~d}$ vortex method, J. Comp. Phys., Vol.152, pp.305-345.

Maruyama, T. and Morikawa, H., 1994, Numerical simulation of wind fluctuation conditioned by experimental data in turbulent boundary layer, Proc. $13^{\text {th }}$ Symp. On Wind Eng., pp.573-578. (in Japanese)

Ogami, T. and Akamatsu, T., 1991, Viscous flow simulation using the discrete vortex method - the diffusion velocity method, Computers \& Fluids, Vol.19, pp.433-441.

Tennekes, H. and Lumley, J. L., 1999, A First Course in Turbulence, $17^{\text {th }}$ edn., MIT Press, Cambridge, pp. 273.

Totsuka, Y. and Obi, S., 2000, Turbulent flow analysis by a three dimensional vortex method (estimation of the dissipation rate), Proceedings of $14^{\text {th }}$ Symposium of Computational Fluid Dynamics, E09-4 (in Japanese). 